\title{
Performance Analysis of Microcellular Mobile Radio Systems with Selection Combining in the Presence of Arbitrary Number of Cochannel Interferences
}

\author{
Nikola M. SEKULOVIĆ, Mihajlo Č. STEFANOVIĆ, Dragan Lj. DRAČA, \\ Aleksandra S. PANAJOTOVIĆ, Dušan M. STEFANOVIĆ \\ Faculty of Electronic Engineering, University of Nis \\ str. Aleksandra Medvedeva nr.14, 18000 Nis, Serbia \\ nikola.sekulovic@elfak.rs,mihajlo.stefanovic@elfak.ni.ac.rs,dragan.draca@elfak.ni.ac.rs, \\ aleksandra.panajotovic@elfak.ni.ac.rs,dusan.stefanovic@itcentar.rs
}

\begin{abstract}
In this paper, the performance of dual selection combining (SC) receiver in communications systems with a Rician desired signal affected by multiple Nakagami- $m$ cochannel interferences (CCIs) is studied. This investigation is important since such environment seems to be the most realistic in microcellular radio communications systems. The performance analysis includes the channel correlation effect since in practice diversity is usually applied in small terminals so antenna elements can not be placed sufficiently apart to achieve independent fading channels. With assumption that CCIs are mutually independent and identically distributed, analytical expressions for the probability density function (PDF) and cumulative distribution function (CDF) of dual SC output signal-to-interference ratio (SIR) are derived and used to investigate important system performance measures, such as the average bit error probability (ABEP), channel capacity and outage probability. The proposed mathematical analysis is complemented by various graphically presented numerical results to show the effects of various system's parameters. In addition, the impact of diversity to the microcellular system's performance is also explored.
\end{abstract}

Index Terms - cochannel interference, correlated fading channels, Nakagami-m fading, Rician fading, selection diversity combining

\section{INTRODUCTION}

In planning a cellular mobile radio system, a fundamental requirement is to provide specified quality-of-service $(\mathrm{QoS})$ in combination with high system capacity. Cochannel interference $(\mathrm{CCI})$ as a result of frequency reuse which is essential in increasing cellular radio capacity and multipath fading due to multipath propagation are the main factors limiting system's performance [1]. Methods that address these problems are of great interest. Upgrading transmission reliability and increasing capacity without increasing transmission power and bandwidth can be achieved by using diversity techniques, such as space diversity. Space diversity techniques combine input signals from multiple receive antennas [2]. The most popular ones are maximal-ratio combining (MRC), equal-gain combining (EGC), and selection combining (SC) [3]. MRC and EGC require all or some of the channel state information (fading amplitude, phase, and delay). In addition, a separate receiver chain is needed for each diversity branch which increases its complexity. In opposition to MRC and EGC, SC receiver is much simpler for practical realization because it processes only one of the diversity branches. In general, branch with the highest signal-to-noise ratio (SNR) (or equivalently, with the strongest signal assuming equal noise power among the antennas) is connected to the output. Efficient cellular system designs are interference-limited [2], i.e. the level of the thermal noise is sufficiently low as compared to the level of CCI, so the thermal noise effect may be ignored. In that case, SC receiver can select the branch with the highest signal-to-interference ratio (SIR; SIR-based selection diversity) [4].

Most of the published papers study diversity by considering the effect of only the strongest CCI [4]-[14] assuming that the remaining CCIs can be ignored. In general, the performance analysis of cellular mobile radio system should include the influence of multiple CCIs from two or more distant cells [15]-[19]. In medium and large cell systems, both the desired signal and CCIs usually experience the same severity of fading. This means that they can be modeled by the same fading statistics [15]. The spectrum utilization efficiency may be improved by decreasing the size of cells. In a microcellular environment, CCIs usually experience significantly deeper fading than the desired signal. Therefore, different fading models or the same fading statistics but with different parameters, i.e. different amounts of fading for the desired signal and CCIs, have to be used [16]. The desired signal usually experiences Rician fading since a line-of-sight (LoS) path between transmitter and receiver within a microcell may exist [17], [20]. CCIs from distant cells may be modeled by Rayleigh statistics [18]. However, Nakagami- $m$ distribution takes the Rayleigh distribution as special case. In addition, Nakagami$m$ statistics can be used to model fading conditions that are more or less severe than those modeled by Rayleigh statistics. It is reason why Nakagami- $m$ distribution is more appropriate to model CCIs than Rayleigh. The Rician/Nakagami- $m$ model (the desired signal is modeled with Rician distribution while CCIs are modeled using Nakagami- $m$ distribution) for microcellular system is introduced in [19]. But, to the best of the authors' knowledge, performance analysis of diversity system in the above proposed environment is not available in the open 
technical literature.

In this paper, dual branch SC diversity receiver, as one of the most used in wireless communications systems, in correlative Rician/Nakagami- $m$ fading environment is considered. Independent fading assumes antenna elements be placed sufficiently far apart, which is not always realized in practice, especially in small terminals. Therefore, performance analysis of diversity system over correlated fading channels is important from both theoretical and practical viewpoint. Analytical expression for the probability density function (PDF) of SIR at the output of the $\mathrm{SC}$ receiver is derived and used for the average bit error probability (ABEP) and channel capacity analysis. The outage probability, as one more important performance metric, is obtained, too. Numerical results are presented graphically to show the effects of both various system's parameters and diversity to the system's performance.

\section{SYSTEM AND CHANNEL MODEL}

We consider a microcellular mobile radio system with dual SIR-based SC receiver. Due to insufficient antennae spacing, desired signal envelopes experience correlative Rician fading with the joint PDF [21, eq. (4)]

$$
\begin{aligned}
& p_{A_{1} A_{2}}\left(A_{1}, A_{2}\right)=\frac{A_{1} A_{2}(K+1)^{2}}{\beta^{2}\left(1-r^{2}\right)} \\
& \times \exp \left(-\frac{\left(A_{1}^{2}+A_{2}^{2}\right)(K+1)+4 K \beta(1-r)}{2 \beta\left(1-r^{2}\right)}\right) \\
& \times \sum_{i=0}^{\infty} \varepsilon_{i} I_{i}\left(\frac{A_{1} A_{2} r(K+1)}{\beta\left(1-r^{2}\right)}\right) I_{i}\left(\frac{A_{1}}{1+r} \sqrt{\frac{2 K(K+1)}{\beta}}\right) \\
& \times I_{i}\left(\frac{A_{2}}{1+r} \sqrt{\frac{2 K(K+1)}{\beta}}\right), \quad \varepsilon_{i}= \begin{cases}1, & i=0 \\
2, & i \neq 0\end{cases}
\end{aligned}
$$

where $K$ is the Rician factor defined as the ratio of the signal power in the dominant component over the scattered power, $r$ is the branch correlation coefficient, $\beta=\overline{A_{1}^{2}} / 2=\overline{A_{2}^{2}} / 2$ is the average desired signal power and $I_{i}(\cdot)$ is the modified Bessel function of the first kind and $i$ th order.

The desired signal is corrupted by arbitrary number $(n)$ of CCIs. It is assumed that CCIs are mutually independent and identically distributed (i.i.d.) Nakagami- $m$ random variables with the same fading parameter and the same average power $\Omega=\overline{r_{l, j}{ }^{2}}, l=1,2, j=\overline{1, n}$. The latter assumption is not unreasonable in practical applications, since CCIs originate from approximately the same distance from the base station [22]. Let $R_{l}^{2}=r_{l, 1}{ }^{2}+r_{l, 2}{ }^{2}+\ldots+r_{l, n}{ }^{2}, l=1,2$ be the power sums of CCIs on $l$ th diversity branch. The joint PDF of $R_{1}$ and $R_{2}$ is [23, eq. (130)]

$$
\begin{aligned}
& p_{R_{1} R_{2}}\left(R_{1}, R_{2}\right)=\frac{4\left(R_{1} R_{2}\right)^{n m}}{\Gamma(n m)\left(1-r^{2}\right) r^{n m-1} \Omega^{n m+1}} \\
& \times \exp \left(-\frac{R_{1}{ }^{2}+R_{2}{ }^{2}}{\Omega\left(1-r^{2}\right)}\right) I_{n m-1}\left(\frac{2 r R_{1} R_{2}}{\Omega\left(1-r^{2}\right)}\right)
\end{aligned}
$$

where $m$ is the fading severity parameter $(m \geq 0.5)$.

Instantaneous values of SIR on first and second diversity branch can be defined as $\mu_{1}=A_{1}{ }^{2} / R_{1}{ }^{2}$ and $\mu_{2}=A_{2}{ }^{2} / R_{2}^{2}$, respectively. The joint PDF of these random variables is $p_{\mu_{1} \mu_{2}}\left(\mu_{1}, \mu_{2}\right)=\frac{1}{4 \sqrt{\mu_{1} \mu_{2}}} \int_{0}^{\infty} \int_{0}^{\infty} R_{1} R_{2} p_{A_{1} A_{2}}\left(R_{1} \sqrt{\mu_{1}}, R_{2} \sqrt{\mu_{2}}\right)$

$\times p_{R_{1} R_{2}}\left(R_{1}, R_{2}\right) d R_{1} d R_{2}$.

Substituting (1) and (2) in (3) and using the infinite-series representation of the modified Bessel function [24, eq. (9.610)] and [25, eq. (3.326/2)], the joint PDF of input instantaneous SIRs can be expressed as

$$
\begin{aligned}
& p_{\mu_{1} \mu_{2}}\left(\mu_{1}, \mu_{2}\right)=\frac{\exp \left(-\frac{2 K}{1+r}\right)}{\Gamma(n m)} \sum_{i, j, l, s, p=0}^{\infty} \varepsilon_{i} \alpha^{2(i+j+1)+l+s} \\
& \times \frac{K^{i+l+s} r^{i+2(j+p)}\left(1-r^{2}\right)^{i+l+s+n m+1}}{(1+r)^{2(i+l+s)}} \\
& \times \frac{\Gamma(i+j+l+n m+p+1) \Gamma(i+j+s+n m+p+1)}{j ! l ! s ! p ! \Gamma(i+j+1) \Gamma(i+l+1) \Gamma(i+s+1) \Gamma(n m+p)} \\
& \times \frac{\mu_{1}^{i+j+l} \mu_{2}^{i+j+s}}{\left(\alpha \mu_{1}+1\right)^{i+j+l+n m+p+1}\left(\alpha \mu_{2}+1\right)^{i+j+s+n m+p+1}}
\end{aligned}
$$

where the average SIR is defined here as $\gamma=2 \beta / \Omega$, $\alpha=(K+1) / \gamma$ and $\Gamma(\cdot)$ is the gamma function.

The corresponding bivariate CDF is by definition

$$
F_{\mu_{1} \mu_{2}}\left(\mu_{1}, \mu_{2}\right)=\int_{0}^{\mu_{1}} \int_{0}^{\mu_{2}} p_{\mu_{1} \mu_{2}}\left(x_{1}, x_{2}\right) d x_{1} d x_{2} \text {. }
$$

Substituting (4) in (5) and after some straightforward mathematical manipulations, integrals can be solved with the use of [25, eq. (3.194/1)] resulting in

$$
\begin{aligned}
& F_{\mu_{1} \mu_{2}}\left(\mu_{1}, \mu_{2}\right)=\frac{\exp \left(-\frac{2 K}{1+r}\right)}{\Gamma(n m)} \sum_{i, j, l, s, p=0}^{\infty} \varepsilon_{i} \alpha^{2(i+j+1)+l+s} \\
& \times \frac{K^{i+l+s} r^{i+2(j+p)}\left(1-r^{2}\right)^{i+l+s+n m+1} \mu_{1}^{i+j+l+1} \mu_{2}^{i+j+s+1}}{(1+r)^{2(i+l+s)} j ! l ! s ! p !(i+j+l+1)(i+j+s+1)} \\
& \times \frac{\Gamma(i+j+l+n m+p+1) \Gamma(i+j+s+n m+p+1)}{\Gamma(n m+p) \Gamma(i+j+1) \Gamma(i+l+1) \Gamma(i+s+1)} \\
& \times{ }_{2} F_{1}\left(i+j+l+n m+p+1, i+j+l+1 ; i+j+l+2 ;-\alpha \mu_{1}\right) \\
& \times{ }_{2} F_{1}\left(i+j+s+n m+p+1, i+j+s+1 ; i+j+s+2 ;-\alpha \mu_{2}\right)
\end{aligned}
$$

where ${ }_{2} F_{1}(a, b ; c ; d)$ is the Gaussian hypergeometric function.

$\mathrm{SC}$ receiver chooses the branch with the highest SIR, i.e. $\mu_{S C}=\max \left\{\mu_{1}, \mu_{2}\right\}$. Applying the concepts of probability, the expression for the PDF of $\mu_{S C}$ can be obtained as

$$
p_{\mu_{S C}}(\mu)=\int_{0}^{\mu} p_{\mu_{1} \mu_{2}}\left(\mu, \mu_{2}\right) d \mu_{2}+\int_{0}^{\mu} p_{\mu_{1} \mu_{2}}\left(\mu_{1}, \mu\right) d \mu_{1}
$$

which by using [25, eq. (3.194/1)] yields

$$
\begin{aligned}
& p_{\mu_{S C}}(\mu)=\frac{\exp \left(-\frac{2 K}{1+r}\right)}{\Gamma(n m)} \sum_{i, j, l, s, p=0}^{\infty} \varepsilon_{i} \alpha^{2(i+j+1)+l+s} \\
& \times \frac{K^{i+l+s} r^{i+2(j+p)}\left(1-r^{2}\right)^{i+l+s+n m+1} \mu^{2(i+j)+l+s+1}}{(1+r)^{2(i+l+s)}} \\
& \times \frac{\Gamma(i+j+l+n m+p+1) \Gamma(i+j+s+n m+p+1)}{j ! l ! s ! p ! \Gamma(i+j+1) \Gamma(i+l+1) \Gamma(i+s+1) \Gamma(n m+p)}
\end{aligned}
$$




$$
\begin{aligned}
& \times\left[\frac{{ }_{2} F_{1}(i+j+l+n m+p+1, i+j+l+1 ; i+j+l+2 ;-\alpha \mu)}{(i+j+l+1)(\alpha \mu+1)^{i+j+s+n m+p+1}}\right. \\
& \left.+\frac{{ }_{2} F_{1}(i+j+s+n m+p+1, i+j+s+1 ; i+j+s+2 ;-\alpha \mu)}{(i+j+s+1)(\alpha \mu+1)^{i+j+l+n m+p+1}}\right] .
\end{aligned}
$$

The CDF of the instantaneous SIR at the output of SC combiner can be easily obtained from (6) as

$F_{\mu_{S C}}(\mu)=F_{\mu_{1} \mu_{2}}(\mu, \mu)$.

\section{PERFORMANCE ANALYSIS}

\section{A. Average Bit Error Probability}

An important and widely accepted performance measure for diversity systems is the ABEP. In digital communications systems, for a given modulation scheme, ABEP can be obtained by averaging the conditional bit error probability over the PDF of the receiver output SIR. For noncoherent binary differential phase-shift keying (BDPSK) modulation, the ABEP is formulated as

$\overline{P_{e}}=\int_{0}^{\infty} 0.5 \exp (-\mu) p_{\mu_{S C}}(\mu) d \mu$.

In Fig. 1, the ABEP is plotted versus $\gamma$ for different correlation coefficients and for several values of the Rician factor and Nakagami parameter which describe fading severity of the desired signal and CCIs, respectively. It is assumed that desired signal competes with two CCIs. The system shows better performance for lower values of the correlation coefficient, i.e. for larger spatial separation between the received antennas in terminals. If the correlation is too high, then deep fades in the branches will occur simultaneously resulting in low improvement degree of considered space diversity system. In practice, the maximum theoretical diversity gain cannot be achieved because it is not likely to realize independent channels in diversity system applied on small terminals. It can be also observed that the ABEP improves when the Rician factor increases. This happens because a higher value of the Rician factor means that the desired signal contains a large LoS component and a small diffuse-scattered component, i.e. the desired signal suffers less from severe fading. On the other hand, lower values of the Nakagami parameter mean deep

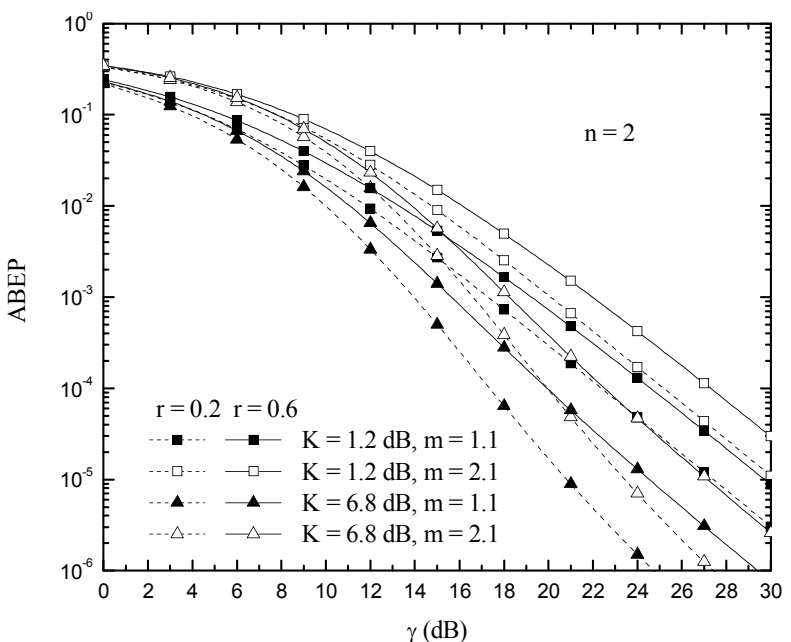

Fig. 1. ABEP of BDPSK system versus input average SIR for several values of the correlation coefficient, Rician factor and Nakagami parameter. fading behavior for the CCIs which lead to the system performance enhancement.

Presented expression for the ABEP converges rapidly enabling great accuracy of the evaluated and graphically presented numerical results. Table I summarizes the number of terms in every of five sums that need to be summed to achieve accuracy at the fourth significant digit after the truncation of the infinite series for a range of values for $K$, $m, r$, and $\gamma$. We observe that the number of terms increases as $K, m$ or $r$ increases.

TABLE I. NUMBER OF TERMS THAT NEED TO BE SUMMED IN EXPRESSION FOR THE ABEP TO ACHIEVE ACCURACY AT THE 4TH SIGNIFICANT DIGIT ( $n=2)$

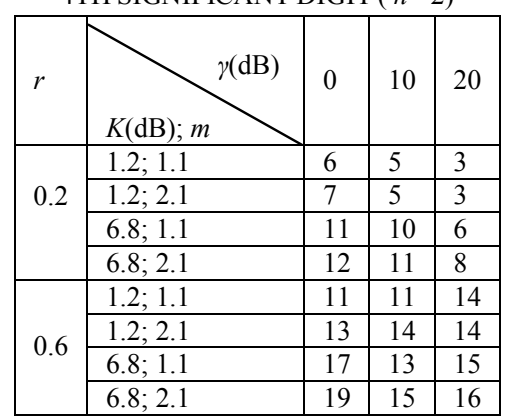

\section{B. Channel Capacity}

Channel capacity is an important performance measure in design of future mobile radio cellular systems since it provides an upper bound of maximum transmission rate. The average channel capacity is defined as

$\bar{C}=B W \int_{0}^{\infty} \log _{2}(1+\mu) p_{\mu_{S C}}(\mu) d \mu$

where $B W$ is channel bandwidth

In Fig. 2, the normalized average channel capacity is plotted as a function of $\gamma$. The influence of number of CCIs on system performance was in focus. As it was expected, system performance degrades with the increase of number of CCIs. However, the gap among the curves decreases as number of CCIs increases. If the relative decreasing of the average channel capacity due to increase of number of CCIs is defined as

$$
\Delta_{n, n+1}=\left[1-\frac{(\bar{C} / B W)_{n+1}}{(\bar{C} / B W)_{n}}\right] \cdot 100 \%,
$$

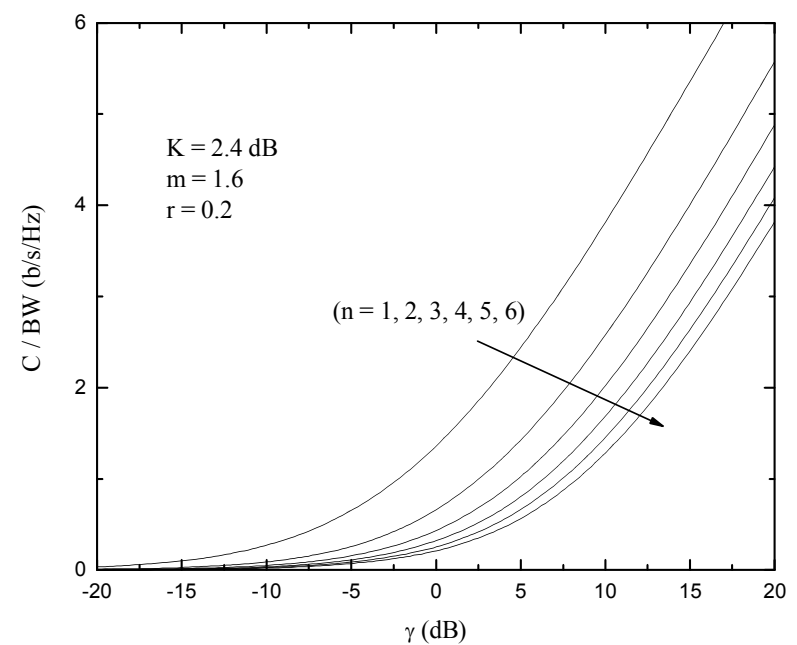

Fig. 2. Normalized average channel capacity versus input average SIR for arbitrary number of CCIs. 
for $K=2.4 \mathrm{~dB}, r=0.2, m=1.6$, and $\gamma=0 \mathrm{~dB}$, we obtained $\Delta_{1,2}=51.28 \%, \Delta_{2,3}=34.4 \%, \Delta_{3,4}=25.77 \%, \Delta_{4,5}=20.56 \%$ and $\Delta_{5,6}=17.09 \%$. Therefore, the influence of number of CCIs on relative average channel capacity degradation reduces with increment of that number. Results in Table II indicate that expression (11) is very operable for presented analysis because very few terms are required for the desired accuracy.

TABLE II. NUMBER OF TERMS THAT NEED TO BE SUMMED IN EXPRESSION FOR THE AVERAGE CHANNEL CAPACITY TO ACHIEVE ACCURACY AT THE 4TH SIGNIFICANT DIGIT

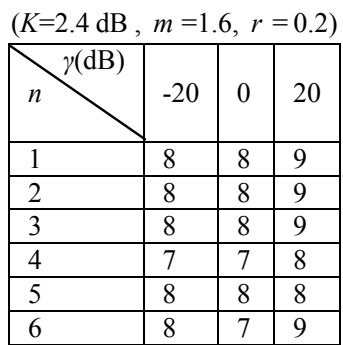

\section{Outage Probability}

Another useful performance measure in wireless communications systems design, especially for the cases when CCI is present, is the outage probability. In the interference-limited environment, the outage probability is defined as the probability that the combiner's output SIR falls below a given outage threshold $\mu_{t h}$, also known as a protection ratio which is determined by expected QoS

$$
P_{\text {out }}=P_{R}\left(\mu<\mu_{t h}\right)=\int_{0}^{\mu_{t h}} p_{\mu_{S C}}(\mu) d \mu=F_{\mu_{S C}}\left(\mu_{t h}\right) \text {. }
$$

Table III shows that presented infinite-series expression converges rapidly, and thus, it can efficiently be used in the following outage probability analysis.

\section{TABLE III. NUMBER OF TERMS THAT NEED TO BE SUMMED IN EXPRESSION FOR THE OUTAGE PROBABILITY TO ACHIEVE ACCURACY AT THE 4TH SIGNIFICANT DIGIT}

\begin{tabular}{|c|c|c|c|c|}
\hline \\
\hline$r$ & ${ }_{n} \mu_{\mathrm{th}}(\mathrm{dB})$ & -20 & 0 & 20 \\
\hline \multirow{3}{*}{0.2} & 1 & 3 & 6 & 7 \\
\hline & 2 & 4 & 5 & 7 \\
\hline & 4 & 3 & 5 & 7 \\
\hline \multirow{3}{*}{0.6} & 1 & 13 & 10 & 14 \\
\hline & 2 & 14 & 12 & 14 \\
\hline & 4 & 16 & 15 & 16 \\
\hline
\end{tabular}

As we mentioned earlier, in order to increase channel capacity, system planners introduce smaller cells with base stations transmitting at much lower power. This also means that a distance between the base stations using the same channel frequencies is smaller resulting in a larger number of CCIs. It is a good idea to compare cases with different number of CCIs, in the light of QoS, under assumption that the total average power of CCIs is constant. In first case, there is one CCI with the average power $\Omega$. This case seems the most appropriate for conventional designed cellular mobile radio systems in which the service area is subdivided into larger cells with high-powered base stations. In second case, there are two CCIs, each with the average power $0.5 \Omega$, while in third case, there are four CCIs, each with the average power $0.25 \Omega$. The presence of more and weaker CCIs is characteristic for microcellular mobile radio communications systems. In Fig. 3, the outage probability curves for these cases are presented. For $\mu_{t h}<0 \mathrm{~dB}$ (Fig. $3 a$ ), the influence of number of CCIs on the outage performance is negligible, especially for lower values of the correlation coefficient. In this range of $\mu_{t h}$, for higher values of the correlation coefficient, system shows a little better performance in presence of larger number and weaker CCIs. On the opposite, for $\mu_{t h}>0 \mathrm{~dB}$ (Fig. 3b), system performance is better in cases with smaller number but stronger CCIs. Having in mind that the outage threshold is greater than $10 \mathrm{~dB}$ in practice, we can make conclusion that increasing of number of lower powered transmitters operating on the same frequency as the wanted transmitter degrades QoS.

The fact that space diversity is an effective and widely employed technique in digital communication receivers for improving the overall wireless systems performance gives idea to investigate diversity gain of dual SC receiver in Rician/Nakagami- $m$ environment. For that purpose, in the appendix, we derive the analytical expressions for the outage probability for a microcellular

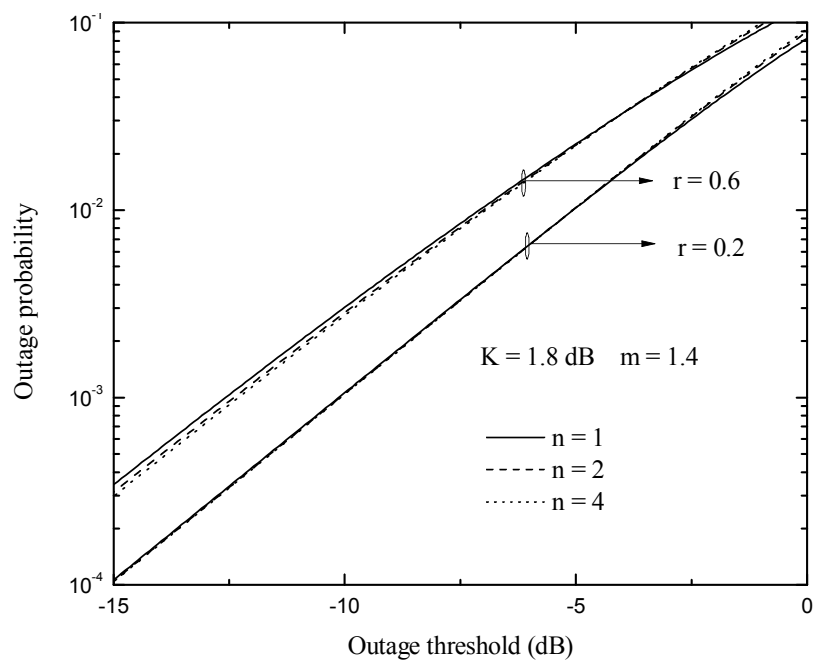

a)

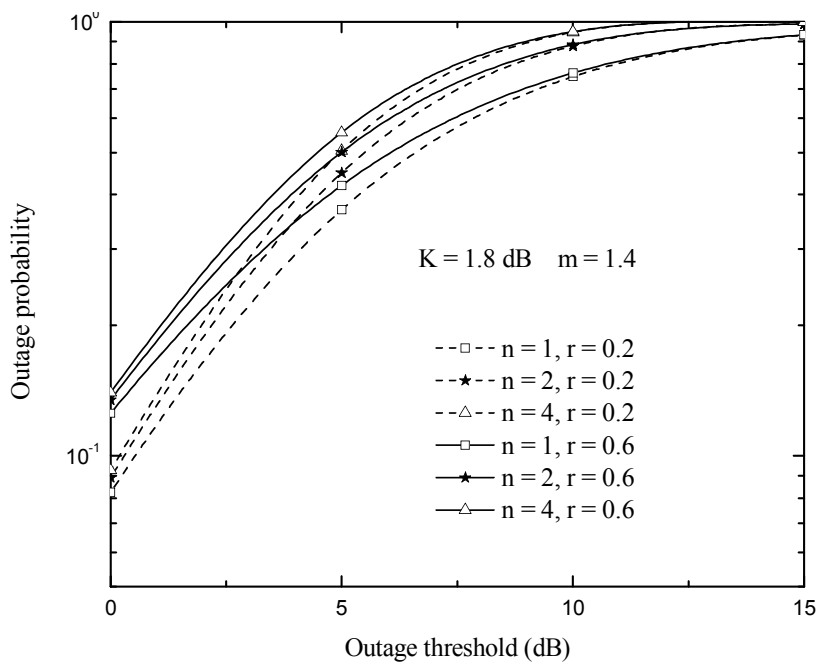

b)

Fig. 3. Outage probability as a function of the outage threshold for differen system parameters. 


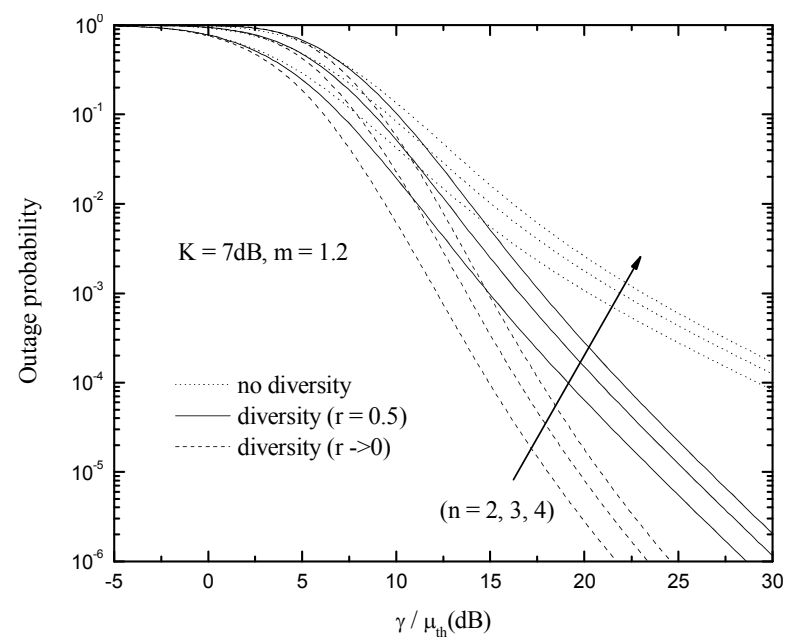

Fig. 4. Diversity effect on the outage probability in Rician/Nakagami- $m$ environment.

environment without diversity. The outage performance versus normalized parameter $\gamma / \mu_{t h}$ is shown in Fig. 4 for the case with and without diversity. For comparison purposes, the curves for the system with uncorrelated fading $(r \rightarrow 0)$ are also included as a special case for the best performance. System performance enhancement due to use of diversity is obvious. For the limiting case of independent fading channels, at an outage probability of $10^{-2}$, the diversity gain is about $4.2 \mathrm{~dB}$, while the gain is about $8.3 \mathrm{~dB}$ at an outage probability of $10^{-3}$. The diversity gain here is defined as the reduction in the average SIR compared with the case of no diversity. However, as we mentioned earlier, in practice due to insufficient spacing between antennas, fading channels are correlated. For $r=0.5$, at an outage probability of $10^{-2}$ and $10^{-3}$, the diversity gain is about 2.6 $\mathrm{dB}$ and $5.4 \mathrm{~dB}$, respectively. The results indicate that the diversity gain is larger for lower values of the outage probability. It is also interesting to note that the number of CCIs has not influence on the diversity gain.

\section{CONCLUSION}

In a cellular mobile radio system, the main factors limiting performance are CCIs from neighboring cells and multipath fading. Dual SC receiver over correlated Rician fading channels in the presence of arbitrary number of Nakagami- $m$ CCIs was studied in this paper. This scenario characterizes the microcellular environment. In cases in which capacity is an issue, it is very effective to introduce microcellular structures. This confirms relevancy and actuality of the presented analysis. The analytical expressions for the PDF and CDF of the instantaneous SIR at the output of dual SC receiver were derived. These expressions converge rapidly, and thus, they can be efficiently used to analytical study several performance criteria, such as the ABEP, outage probability and average channel capacity. Numerical results were graphically presented, showing that system performance improves with increase of the Rician factor and with decrease of the correlation coefficient, Nakagami parameter and number of CCIs. Under assumption that the total average power of CCIs is constant, it was shown that increasing of number of CCIs for values of practical interest degrades the outage performance. In other words, systems designers have to make compromise between capacity and QoS. In addition, an improvement in outage probability due to diversity was also observed.

\section{APPENDIX}

In this appendix, useful expressions for the PDF and CDF of SIR in microcellular environment without diversity are derived. The desired signal follows the Rician distribution with the PDF given by [26, eq. (4)]

$$
p_{A}(A)=\frac{2 A(K+1)}{\beta_{1}} \exp \left(-K-\frac{K+1}{\beta_{1}} A^{2}\right) I_{0}\left(2 \sqrt{\frac{K(K+1)}{\beta_{1}}} A\right)
$$

where the average power is defined here as $\beta_{1}=\overline{A^{2}}$. If $R^{2}=r_{1}^{2}+r_{2}^{2}+\ldots+r_{n}^{2}$ is the power sum of Nakagami- $m$ interferences, the PDF of $R$ is [23, eq. (71)]

$$
p_{R}(R)=\frac{2 m^{n m} R^{2 n m-1}}{\Gamma(n m) \Omega^{n m}} \exp \left(-\frac{m}{\Omega} R^{2}\right) .
$$

Applying the well-known concepts of probability, the PDF and CDF of the instantaneous SIR are respectively

$$
p_{\mu}(\mu)=\frac{\exp (-K)}{\Gamma(n m)} \sum_{i=0}^{\infty} \frac{K^{i} \alpha^{i+1} \mu^{i} \Gamma(i+n m+1)}{(i !)^{2} m^{i+1}\left(1+\frac{\alpha \mu}{m}\right)^{i+n m+1}}
$$

and

$$
\begin{aligned}
& F_{\mu}(\mu)=\frac{\exp (-K)}{\Gamma(n m)} \sum_{i=0}^{\infty} \frac{K^{i} \alpha^{i+1} \mu^{i+1} \Gamma(i+n m+1)}{(i+1) ! \Gamma(i+1) m^{i+1}} \\
& \times_{2} F_{1}\left(i+n m+1, i+1 ; i+2 ;-\frac{\alpha \mu}{m}\right)
\end{aligned}
$$

where $\gamma$ is the average SIR defined as $\gamma=\beta_{1} / \Omega$. These expressions are derived in order to investigate the impact of diversity on the microcellular system's performance. The outage probability can be simply calculated as

$P_{\text {out }}=F_{\mu}\left(\mu_{t h}\right)$.

An alternative exact formulation of the outage probability in mobile radio cellular system with a Rician signal in the presence of arbitrary number of CCIs with arbitrary parameters is presented in [19]. The derived formula can be calculated numerically using GaussHermitte method with the desired accuracy. But, in the case of i.i.d. CCIs, it is more operable to use the above presented analytical expression for the outage probability. Furthermore, the PDF expression obtained in this Appendix can be used for ABEP or channel capacity analysis.

\section{REFERENCES}

[1] J. D. Parsons, The Mobile Radio Propagation Channels, 2nd ed. New York: Wiley, 2000.

[2] A. Goldsmith, Wireless Communications, Cambridge University Pres: New York, 2005.

[3] M. K. Simon and M.-S. Alouini, Digital Communication over Fading Channels, 1st ed. New York: Wiley, 2000.

[4] S. Okui, "Effects of SIR selection diversity with two correlated branches in the m-fading channel," IEEE Trans. Commun., vol. 48, no. 10, pp. 1631-1633, Oct. 2000.

[5] A. S. Panajotović, M. Č. Stefanović, D. Lj. Drača, and N. M. Sekulović, "Average level crossing rate of dual selection diversity in correlated Rician fading with Rayleigh cochannel interference," IEEE Commun. Lett., vol. 14, no. 7, pp. 1-3, Jul. 2010.

[6] J. Winters, "Optimum combining in digital mobile radio with cochannel interference," IEEE J. Sel. Areas in Commun., vol. 2, no. 4, pp. 528-539, Jul. 1984 
[7] G. K. Karagiannidis, "Performance analysis of SIR-based dua selection diversity over correlated Nakagami-m fading channels,' IEEE Trans. Veh. Technol., vol. 52, no. 5, pp. 1207-1216, Sept. 2003.

[8] M. Č. Stefanović, D. M. Milović, A. M. Mitić, and M. M. Jakovljević, "Performance analysis of system with selection combining over correlated Weibull fading channels in the presence of cochannel interference," Int. J. Electron. Commun. (AEÜ), vol. 62, no. 9, pp. 695-700, Oct. 2008.

[9] A. S. Panajotović, M. Č. Stefanović, and D. Lj. Drača, "Performance analysis of system with selection combining over correlated Rician fading channels in the presence of cochannel interference," Int. J. Electron. Commun. (AEÜ), vol. 63, no. 12, pp. 1061-1066, Dec. 2009.

[10] M. Č. Stefanović, D. Lj. Drača, A. S. Panajotović, and N. M Sekulović, "Performance analysis of system with L-branch selection combining over correlated Weibull fading channels in the presence of cochannel interference," Int. J. Commun. Syst., vol. 23, no. 2, pp. 139-150, Feb. 2010.

[11] A. S. Panajotović, M. Č. Stefanović, and D. Lj. Drača, "Effect of microdiversity and macrodiversity on average bit error probability in shadowed fading channels in the presence of interference," ETRI J., vol. 31, no. 5, pp. 500-505, Oct. 2009.

[12] D. Aleksić, N. Sekulović, and M. Stefanović, "Outage probability of system with selection combining over correlated Weibull fading channels in the presence of Rayleigh cochannel interference,' Elektron. Elektrotech., vol. 90, no. 2, pp. 7-10, 2009.

[13] P. Spalević, N. Sekulović, Z. Georgios, and E. Mekić, "Performance analysis of SIR-based triple selection diversity over correlated Weibull fading channels," Facta Universitatis: Series Electronics and Energetics, vol. 23, no. 1, pp. 89-98, Apr. 2010.

[14] N. Sekulović, M. Stefanović, D. Denić, and D. Aleksić, "Performance analysis of SINR-based selection diversity over correlated Rayleigh fading channels," IET Commun., accepted for publication, DOI: 10.1049/iet-com.2010.0143.

[15] K. W. Sowerby and A. G. Williamson, "Outage probability calculations for a mobile radio system having multiple Rayleigh interferers," Electron. Lett., vol. 23, no. 11, pp. 600-601, May 1987.
[16] Y.-D. Yao and A. U. H. Sheikh, "Investigations into cochannel interference in microcellular mobile radio systems," IEEE Trans. Veh. Technol., vol. 41, no. 2, pp. 114-123, May 1992.

[17] R. J. C. Bultitude and G. K. Bedal, "Propagation characteristics on microcellular urban mobile radio channels at $910 \mathrm{MHz}$," IEEE J. Sel Areas in Commun., vol. 7, no. 1, pp. 31-39, Jan. 1989.

[18] Y.-D. Yao and A. U. H. Sheikh, "Outage probability analysis for microcell mobile radio systems with cochannel interferers in Rician/Rayleigh fading environment," Electron. Lett., vol. 26, no. 13, pp. 864-866, Jun. 1990.

[19] G. Karagiannidis, S. Kotsopoulos, and C. Georgopoulos, "Outage probability analysis for a Rician signal in L Nakagami-m interferers with arbitrary parameters," J. Commun. Networks, vol. 1, no. 1, pp. 26-30, Mar. 1999

[20] V. V. Milenković, N. M. Sekulović, M. C. Stefanović, and M. B. Petrović, "Effect of microdiversity and macrodiversity on average bit error probability in gamma shadowed Rician fading channels," ETRI J., vol. 32, no. 3, pp. 464-467, Jun. 2010.

[21] M. K. Simon, "Comments on "Infinite-series representations associated with the bivariate Rician distribution and their applications," " IEEE Trans. Commun., vol. 54, no. 8, pp. 1511-1512, Aug. 2006.

[22] A. A. Abu-Dayya and N. C. Beaulieu, "Outage probabilities of cellular mobile radio systems with multiple Nakagami-m interferers," IEEE Trans. Veh. Technol., vol. 40, no. 4, pp. 757-768, Nov. 1991.

[23] M. Nakagami, "The m-distribution - A general formula of intensity distribution of rapid fading," in Statistical Methods in Radio Wave Propagation, Pergamon Press, Oxford, U.K., pp. 3-36, 1960.

[24] M. Abramovitz and I. A. Stegun, Handbook of Mathematical Functions with Formulas, Gaphs, and Mathematical Tables, New York: Dover publications Inc., 1970.

[25] I. S. Gradshteyn and I. M. Ryzhik, Table of Integrals, Series, and Products, 5th ed. New York: Academic, 1994.

[26] A. A. Abu-Dayya and N. C. Beaulieu, "Microdiversity on Rician fading channels," IEEE Trans. Commun., vol. 42, no. 6, pp. 22582267, Jun. 1994 